\title{
Evaluation of Toxicity and Antiangiogenic Activity of Murraya koenigii Leaf Extracts in Zebrafish
}

\author{
Deeksha Dinesh ${ }^{1}$ Dechamma Pandyanda Nanjappa ${ }^{1}$ Nishith Babu ${ }^{1} \quad$ Krithika Kalladka ${ }^{1}$ \\ Gunimala Chakraborty ${ }^{1}$ Anirban Chakraborty ${ }^{1}$
}

${ }^{1}$ Division of Molecular Genetics and Cancer, Nitte University Centre for Science and Education, Nitte

(Deemed to be University), Mangalore, Karnataka, India

\begin{abstract}
Address for correspondence Anirban Chakraborty, PhD, Division of Molecular Genetics and Cancer, Nitte University Centre for Science and Education, Nitte (Deemed to be University), Deralakatte, Mangalore 575018, Karnataka, India (e-mail: anirban@nitte.edu.in).
\end{abstract}

Abstract

Keywords
- Murraya koenigii leaves
- embryo
- toxicity
- zebrafish
- antiangiogenic
activity
- Tg (fli1: GFP)

Introduction This study aimed to evaluate the toxicity and the antiangiogenic activity of Murraya koenigii leaf extracts in zebrafish.

Materials and Methods Crude aqueous and ethanol extracts of M. koenigii leaves were obtained following a standard solvent-based extraction method. The toxicity of these extracts on zebrafish embryonic development was determined and the $\mathrm{LC}_{50}$ values were calculated. Finally, the antiangiogenic activity of the $M$. koenigii leaf extracts was evaluated in a transgenic zebrafish ( $T g$ [fli1: GFP]) that express GFP under the control of fli1, a transcription factor involved in vasculogenesis.

Results The $\mathrm{LC}_{50}$ value of the aqueous extract of $M$. koenigii leaves was much larger (2,450 ppm) compared with that of ethanol extract (9.85 ppm), suggesting a lower toxicity of the aqueous extract. The antiangiogenic activity was evident only in aqueous extract, with significant effects in formation of intersegmental vessels and subintestinal veins in treated $\mathrm{Tg}$ (fli1: GFP) zebrafish embryos.

Conclusion This study demonstrated the biological activity of $M$. koenigii leaf extracts as angiogenesis suppressor, suggesting its therapeutic potential as an angiogenesis inhibitor. Identification of active components from natural sources with potent antiangiogenic activity could lead to the development of anticancer agents.

\section{Introduction}

The increasing demand for chemical diversity in screening programs leads to the identification of therapeutic drugs from natural products, particularly from edible plants. According to the World Health Organization, more than $80 \%$ of the world's population rely on traditional medicine for their primary health care needs. ${ }^{1,2}$ The application of medicinal plants in traditional medical treatments is significant in both developing and industrialized countries., ${ }^{3,4}$

Murraya koenigii (curry tree), a small aromatic plant belonging to the family Rutaceae, is native to India and is used in the Ayurvedic system of medicine. ${ }^{5}$ Leaves, roots, and bark of this plant have been demonstrated as rich sources of carbazole alkaloids with potent biological activities. ${ }^{6}$ Some of the pharmacological effects such as antioxidant, antidiabetic, anti-inflammatory, antitumor, anticancer, and neuroprotective activities have been reported for $M$. koenigii. ${ }^{2,7,8}$ The leaves are rich source of polyphenols, which can inhibit the proteolytic activity of the cancer cell and cause cell death.

Angiogenesis is a complex process involved in the development of new blood vessels from preexisting vasculature, mainly for endothelial cell proliferation, organization, and migration. ${ }^{9,10}$ It plays an important role in tumor progression by stimulating blood vessel growth from nearby capillaries as a prelude to metastasis. Tumor growth and metastasis in cancer states have been reported as angiogenesis dependent. ${ }^{11}$ Persistent unregulated angiogenesis is a hallmark of cancer. ${ }^{12}$ Therefore, blocking angiogenesis is considered as an encouraging strategy in cancer therapy. 
In recent years, zebrafish (Danio rerio) has gained tremendous popularity as an in vivo model in biomedical research due to its several inherent properties. Zebrafish has high fecundity, the embryos are optically transparent, and it has short generation time. The zebrafish is amenable to high throughput screening in drug discovery and it is inexpensive to maintain. The ease of genetic manipulation and the availability of many transgenic lines where the blood vessels are tagged with fluorescent proteins allowing real-time visualization of the process of vasculogenesis make it an ideal system for screening antiangiogenic compounds. ${ }^{13,14}$

In this study, we have obtained crude extracts of $M$. koenigii leaves using two different solvents and determined the toxicity of these compounds. Further, we investigated the antiangiogenic activities of these extracts in transgenic zebrafish embryos ( $\mathrm{Tg}$ [fli1: GFP]) that expressed GFP under the control of fli1, a transcription factor involved in vasculogenesis. The results of this study suggest that $M$. koenigii leaves contain potent angiogenesis inhibitors, which could be further characterized and developed as potent anticancer drugs.

\section{Materials and Methods}

\section{Sample Collection and Extraction}

The M. koenigii leaves were collected from in and around Mangalore. A thorough washing of the collected leaves was done with tap water followed by distilled water. The leaves were then air dried at room temperature for 48 to 72 hours, powdered, and were stored in an airtight container. Solvent extraction was performed following a previously described method..$^{15}$ In brief, $10 \mathrm{~g}$ of dried leaf powder and $100 \mathrm{~mL}$ of the ethanol and water were added separately to $500 \mathrm{~mL}$ conical flask. It was kept in the shaking incubator at $37^{\circ} \mathrm{C}$ for overnight at $110 \mathrm{rpm}$. Following day, the suspension was taken from the incubator and filtered using muslin cloth and was poured into petri plates. These petri plates were kept at room temperature for drying. After drying, the extract was scrapped using spatula and was finely powered. The extract was then transferred into $15 \mathrm{~mL}$ conical tubes, and stored in $4^{\circ} \mathrm{C}$. A 2,500 ppm stock was prepared by dissolving $25 \mathrm{mg}$ extract in $25 \mathrm{~mL}$ solvent. It was than centrifuged at $12,000 \mathrm{rpm}$ for 2 minutes. The filtrate was collected and used for exposure studies. For every trial, fresh stock was prepared and used.

\section{Animal Husbandry and Rearing}

Adult zebrafish were maintained at $28^{\circ} \mathrm{C}$ in a recirculating aquaculture system with 14/10-hour day/night light cycle. Embryos were generated by natural pairwise mating of adult fish. ${ }^{16}$ Breeding boxes were placed into the fish tank overnight. Embryos were collected in the morning and transferred into petri dishes containing embryo media (E3) and subsequently maintained at $28.5^{\circ} \mathrm{C}$.

\section{$\mathbf{L C}_{50}$ Determination}

A semistatic methodology, where media change with renewal of test compound twice a day, was followed. The experiments were performed in triplicates in microtiter plates with 10 embryos in each replicate. The embryos were exposed at 10-hour postfertilization (hpf) and mortality was recorded after 24 hours postexposure. To calculate $\mathrm{LC}_{50}$, Kärber's method ${ }^{17}$ was employed where the concentration at which all the embryos were dead $\left(\mathrm{LC}_{100}\right)$ was considered as the upper limit and the concentration at which all the embryos survived $\left(\mathrm{LC}_{0}\right)$ was considered as the lower limit. Toxicity studies were conducted in accordance with OECD guidelines.

\section{Evaluation of Developmental Toxicity of Aqueous and Ethanol Extracts}

The developmental toxicity was evaluated for each of the concentrations. The experiments were conducted in triplicates with 10 embryos each. The embryos were observed for morphological changes for 5 days with change of water and renewal of test solution twice a day. The developmental stage at first exposure was the shield stage (10 hpf). Four types of toxicological assays, namely, developmental toxicity, cardiotoxicity, neurotoxicity, and hepatotoxicity were performed with observation and recording of lethal and sublethal end points. The effects on development was analyzed on day 1 postfertilization followed by cardiotoxicity on day 2 , neurotoxicity on day 3 , and hepatotoxicity on day 5 . Data from three replicates for each end point for each concentration were recorded using a stereomicroscope at 10 magnification (Leica S9D, Germany) and were compared with the control (unexposed) embryos. The $p$-value was calculated using a $t$-test, and a value of $<0.05$ was considered statistically significant.

\section{Assessment of Antiangiogenic Activity of Aqueous and Ethanol Extract}

To investigate whether the extracts can prevent vasculogenesis in zebrafish embryos, transgenic zebrafish that expresses green fluorescent protein under the control of fli promoter (Tg [fli1: GFP]) was exposed to various concentrations of aqueous and ethanol extracts of $M$. koenigii leaves. Embryos $(n=10)$ in triplicates were exposed to various concentration of the compound at $10 \mathrm{hpf}$ and were observed for the effects on intersegmental vessels (ISVs) and subintestinal veins (SIVs) at 48 and $72 \mathrm{hpf}$. A $10 \mu \mathrm{L}$ of $1 \mathrm{X}$ phenylthiourea was added to all wells to prevent pigmentation. The embryos without exposure of the test compound served as control group. The microtiter plate was incubated at $28.5^{\circ} \mathrm{C}$ for the entire duration. For imaging, the embryos were anesthetized with tricane (final concentration $0.04 \%$ ) and were photographed at 50 magnification using a fluorescent microscope (Leica DM2500, Germany).

\section{Results}

\section{Developmental Toxicity of Aqueous and Ethanol Extracts}

The toxicity of the test compound was determined by calculating the $\mathrm{LC}_{50}$ using the Kärber's method. As shown in - Tables 1 and 2 , the $\mathrm{LC}_{50}$ of the aqueous extract was estimated to be $2,450 \mathrm{ppm}$ and that of ethanol extract was $9.85 \mathrm{ppm}$. The 
results obtained from the exposure of aqueous extract indicated that the crude extract of $M$. koenigii leaves is quite safe without any significant mortality or developmental defects up to $1,000 \mathrm{ppm}$. At $1,500 \mathrm{ppm}$, mortality was observed for

Table 1 Estimation of LC50 for aqueous extract

\begin{tabular}{|l|l|l|l|}
\hline $\begin{array}{l}\text { Concentration } \\
(\mathrm{ppm}) \\
N=30\end{array}$ & $\begin{array}{l}\text { Dose difference } \\
(\mathrm{a})\end{array}$ & $\begin{array}{l}\text { Mean death } \\
(\mathrm{b})\end{array}$ & $(\mathrm{a}) \times(\mathrm{b})$ \\
\hline 4,000 & - & - & - \\
\hline 3,500 & 500 & 9.5 & 4,750 \\
\hline 3,000 & 500 & 8 & 4,000 \\
\hline 2,500 & 500 & 6 & 3,000 \\
\hline 2,000 & 500 & 4.5 & 2,250 \\
\hline 1,500 & 500 & 2.5 & 1,250 \\
\hline 1,000 & 500 & 0.5 & 250 \\
\hline Total & & & 15,500 \\
\hline
\end{tabular}

Note: $\mathrm{LC}_{50}=$ Maximum dose $\left(\sum \mathrm{ab} /\right.$ no. of embryos used $)=4000-$ $(15450 / 10)=2450 \mathrm{ppm}$.

Table 2 Estimation of LC50 for ethanol extract

\begin{tabular}{|l|l|l|l|}
\hline $\begin{array}{l}\text { Concentration } \\
(\mathrm{ppm}) \\
N=30\end{array}$ & $\begin{array}{l}\text { Dose } \\
\text { difference } \\
\text { (a) }\end{array}$ & $\begin{array}{l}\text { Mean death } \\
\text { (b) }\end{array}$ & $(\mathrm{a}) \times(\mathrm{b})$ \\
\hline 12 & - & - & - \\
\hline 11 & 1 & 8 & 8 \\
\hline 10 & 1 & 5 & 5 \\
\hline 9 & 1 & 3.5 & 3.5 \\
\hline 7 & 2 & 2.5 & 2.5 \\
\hline Total & & & 21.5 \\
\hline
\end{tabular}

Note: $\mathrm{LC}_{50}=$ Maximum dose $\left(\sum \mathrm{ab} /\right.$ no. of embryos used $)=12-(24 / 10)=$ $9.85 \mathrm{ppm}$. a few embryos, but it was not statistically significant. For concentrations ranging from 2,000 to 4,000 ppm, though the developmental effects were not evident at day 1 , the effects of the extracts were quite apparent from day 2 onward and at higher concentrations, most of the embryos were dead by day 3 ( - Fig. 1A). Morphological defects including pericardial edema and incomplete yolk absorption were evident at concentrations beyond 2,000 ppm ( - Fig. 1B). Thus, the aqueous extract appeared to be nontoxic at concentrations up to $1,000 \mathrm{ppm}$. In contrast, the ethanol extract appeared to be highly toxic at concentrations beyond $11 \mathrm{ppm}(-$ Fig. 2A). At concentrations between 8 and $10 \mathrm{ppm}$, the embryos were unaffected with a few deaths at day 5 . However, the reduction in number of live embryos was not statistically significant. Severe morphological defects including tail bending, lack of digestive system, and pericardial edema were observed beyond $11 \mathrm{ppm}$. The treated embryos did not survive till $5 \mathrm{dpf}$ (-Fig. 2B).

\section{Cardiotoxicity of Aqueous and Ethanol Extracts}

To check the cardiotoxicity, the heart rate and rhythmicity were analyzed on day 2 postfertilization postexposure to both aqueous and ethanol extracts. The heart beat was calculated based on beats per minute and the values are represented as mean of three independent observations. In case of aqueous extract, there was no change in the heartbeat up to $1,000 \mathrm{ppm}$. However, the heartbeat decreased with increased concentrations and drastic reduction was observed after 3,000 ppm ( - Fig. 3A). In case of ethanol extract, the treated embryos displayed a decrease in heart rate at 8 and $9 \mathrm{ppm}$. Intriguingly, the heartbeat was increased at $11 \mathrm{ppm}$ onward ( - Fig. 3B), suggesting defective cardiac function. However, the looping of the heart was intact for both the extracts, even at higher concentrations, suggesting no effect on structural development of the heart in the treated embryos.

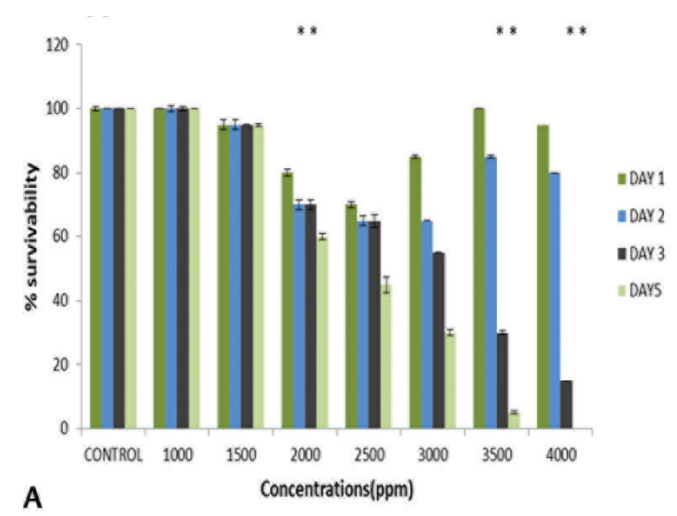

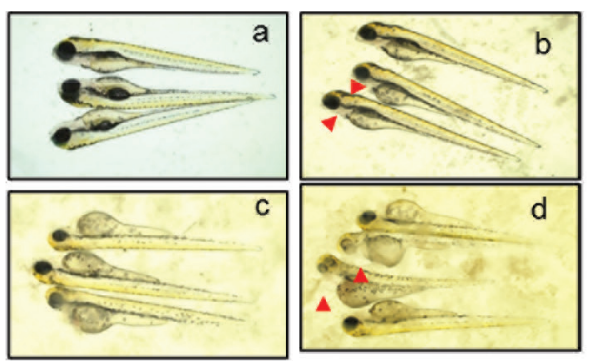

B

Fig. 1 Survival rate and developmental toxicity of zebrafish embryos treated with Murraya koenigii leaf aqueous extract.(A) Survival rates of zebrafish embryos treated with M. koenigii leaf aqueous extract at different days postfertilization. The embryos were exposed at 10 -hour postfertilization (hpf) and the survival rates were recorded at 24, 48, 72 and $120 \mathrm{hpf}$. (B) Representative images of zebrafish embryos at $120 \mathrm{hpf}$ exposed to different concentrations of aqueous extract: a-control, b-2,000 ppm, c-3,000 ppm, and d-3,500 ppm. Morphological anomalies such as incomplete yolk absorption (roundish yolk), pericardial edema (arrowhead), and reduced pigmentation were evident in treated embryos. 


\section{Investigation of Antiangiogenic Property in Murraya koenigii}

To investigate the effects of $M$. koenigii leaf aqueous and ethanol extracts on angiogenesis, a transgenic zebrafish line that expresses GFP under the control of fli1 was used. For determination of antiangiogenic activity, three concentrations below the calculated $\mathrm{LC}_{50}(2,450 \mathrm{ppm}$ for aqueous extract and $9.85 \mathrm{ppm}$ for ethanol extract) that did not show any effects on embryonic development were chosen. As shown in - Fig. 4 , the exposure to the aqueous extract of $M$. koenigii leaves resulted in disruption of the ISV formation leading to defective ISVs (indicated by white box) at 1,000 and 1,500 ppm at $48 \mathrm{hpf}(-$ Fig. $4 \mathrm{~A})$. Although the effects on ISV formation at $48 \mathrm{hpf}$ were marginal, at $72 \mathrm{hpf}$, it was more prominent with conspicuous absence of the proper branching of the ISVs in the treated embryos, particularly at 1,500 and 2,000 ppm, compared with the control ( - Fig. 4B). The aqueous extract also showed a severe effect on the SIV basket formation when observed at $72 \mathrm{hpf}$. As shown in - Fig. 4C, in the control embryos (a), the SIVs were properly formed with well-organized sprouting of the vessels in the form of a mesh-like structure (indicated by white arrow). However, in the treated embryos, there was either ectopic sprouting of SIVs or lack of SIVs suggesting an effect on SIV formation (-Fig. 4C).

In case of ethanol extract, although the branching of the ISVs was not affected, the signal intensity of GFP was lesser in the treated embryos at $48 \mathrm{hpf}$ ( $\mathbf{- F i g}$. 5A). However, at $72 \mathrm{hpf}$, there was no obvious difference between the treated and the

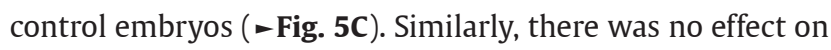

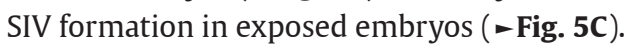

\section{Discussion}

M. koenigii is known for its rich sources of carbazole alkaloids and have been used in traditional medicines because of its various biological properties..$^{18-20}$ The balance between proangiogenic and antiangiogenic factors describes the crucial process of angiogenesis. The imbalances in these factors contribute to impaired angiogenesis leading to vasculature
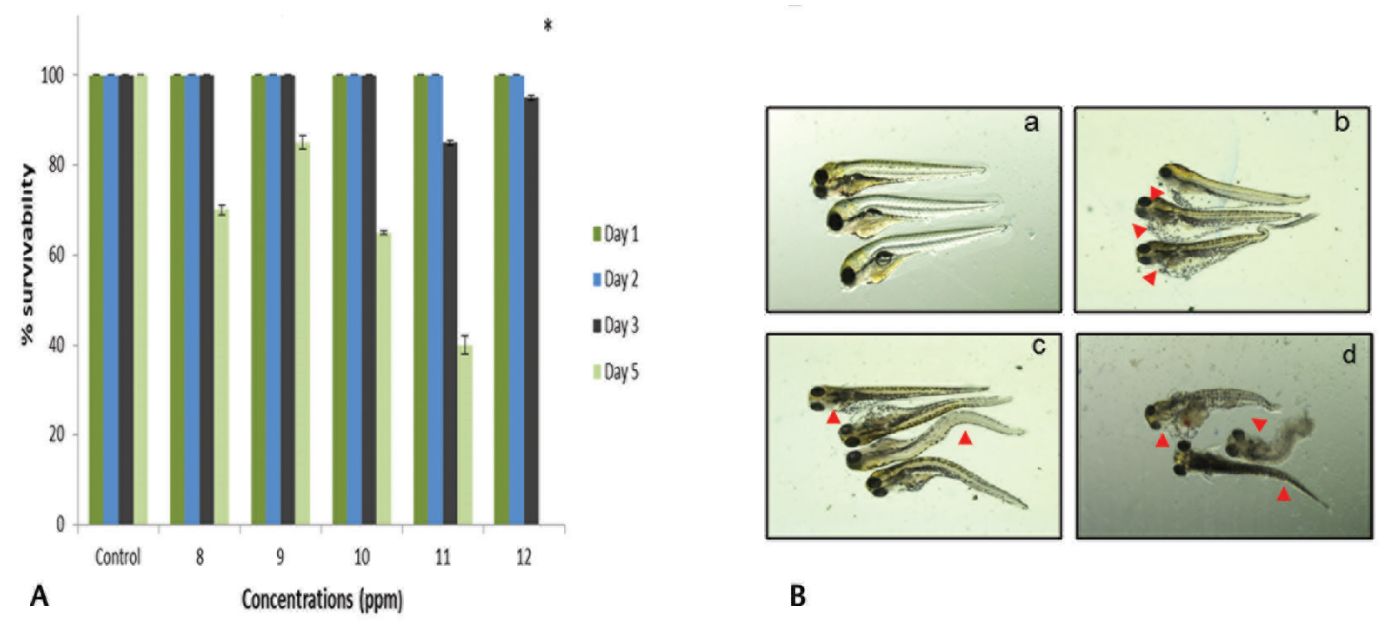

B

Fig. 2 Survival rate and developmental toxicity of zebrafish embryos treated with Murraya koenigii leaf ethanol extract. (A) Survival rates of zebrafish embryos treated with $M$. koenigii leaf ethanol extract at different days postfertilization. The embryos were exposed at 10-hour postfertilization (hpf) and the survival rates were recorded at 24, 48, 72, and $120 \mathrm{hpf}$. (B) Representative images of zebrafish embryos at 120 hpf exposed to different concentrations of ethanol extract: a-control, b-8 ppm, c-10 ppm, and d-12 ppm. Severe developmental effects such as lack of development of digestive tract, tail bending, severe pericardial edema (arrowhead) were evident in treated embryos.
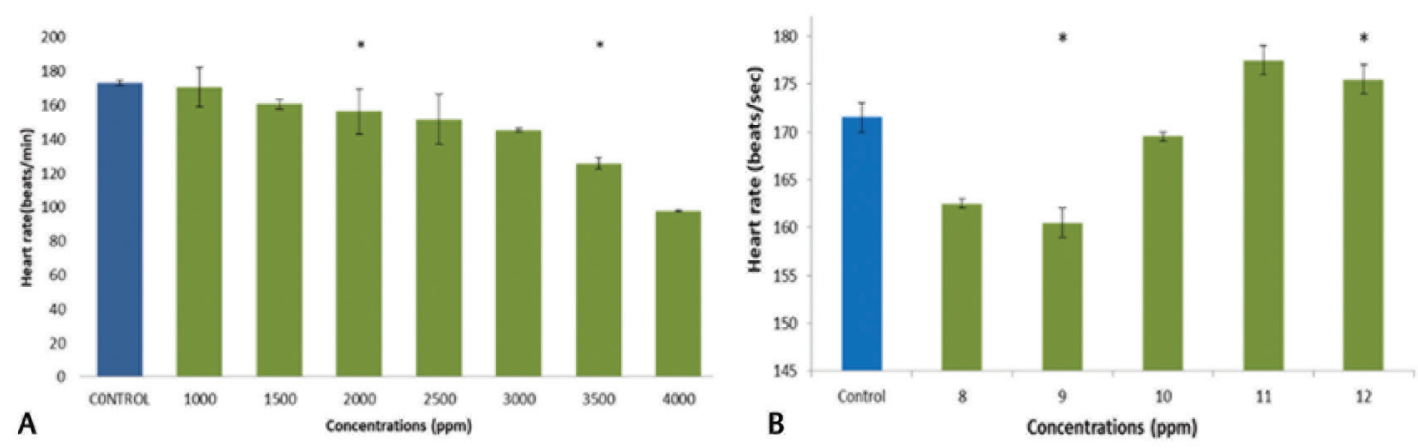

Fig. 3 Effects of Murraya koenigii leaf aqueous and ethanol extracts on heartbeat of zebrafish embryos.(A) Measure of heart beat at 72 -hour postfertilization (hpf) zebrafish embryos treated with different concentration of aqueous extract. (B) Measure of heart beat at 72 hpf zebrafish embryos treated with different concentration of ethanol extract. 

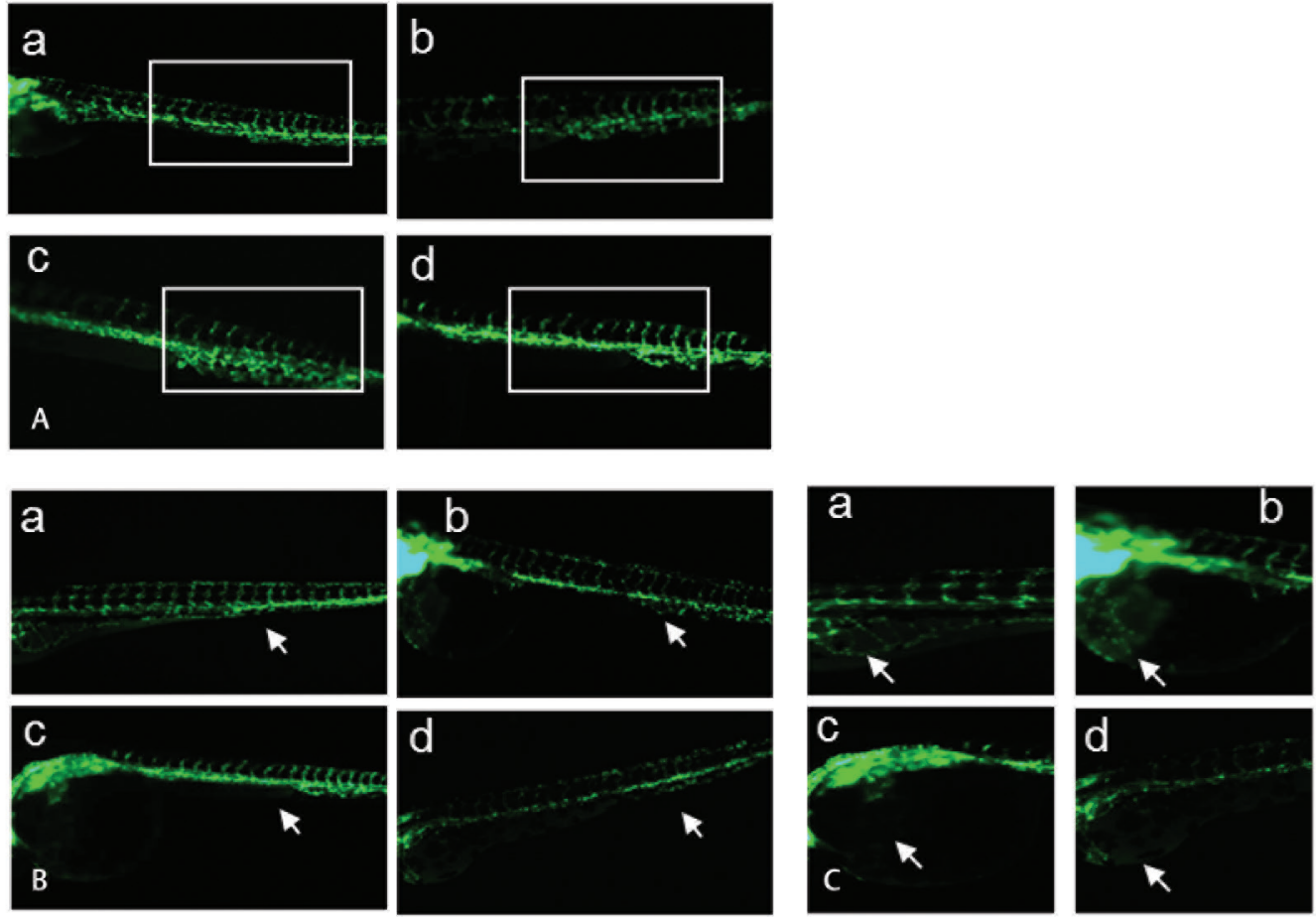

Fig. 4 Antiangiogenic effect of Murraya koenigii leaf aqueous extract on zebrafish transgenic line $T g$ (fli1: GFP).(A, B) Representative images of transgenic zebrafish trunk intersegmental vessels (ISVs) (white box) at 48-hour postfertilization (hpf) (A) and 72 hpf (B) treated with aqueous extract. a-control, b-1,000 ppm, c-1,500 ppm, and d-2,000 ppm. (C) Representative images of subintestinal vein basket (white arrow) of embryos at $72 \mathrm{hpf}$ treated with the extract at different concentration. All figures are lateral views with dorsal to the top and anterior to the left.
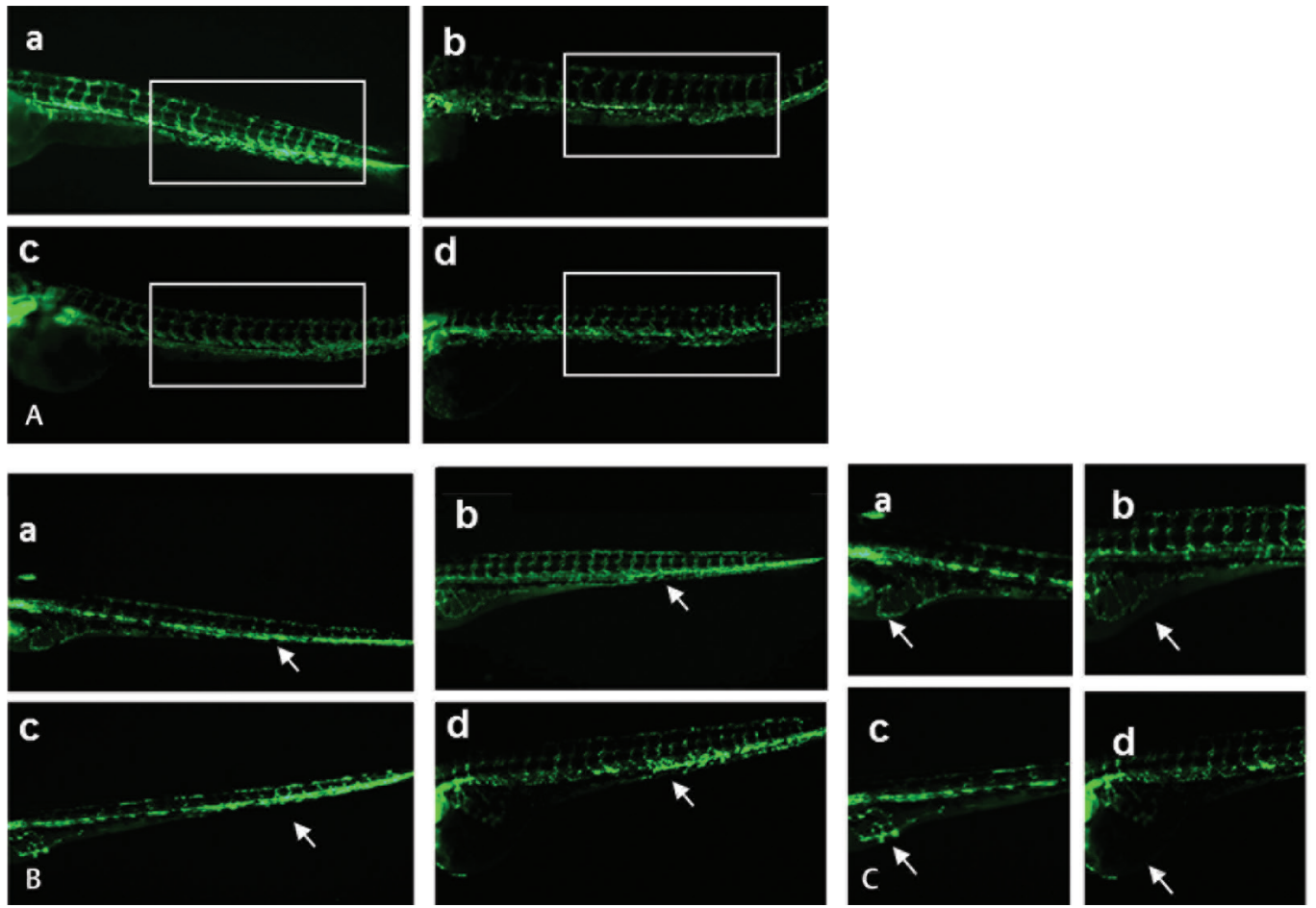

Fig. 5 Angiogenesis in $T g$ (fli1: GFP) zebrafish embryos treated with Murraya koenigii leaf ethanol extract. (A, B) Representative images of zebrafish trunk intersegmental vessels (ISVs) (white box) at 48-hour postfertilization (hpf) (A) and 72 hpf (B) treated with ethanol extract. a-control, b-6 ppm, c-7 ppm, and d-8 ppm. (C) Representative images of subintestinal vein basket (white arrow) of embryos at 72 hpf treated with the extract at different concentration. All figures are lateral views with dorsal to the top and anterior to the left. 
abnormalities which are exacerbated as tumor continues to grow. ${ }^{21,22}$ A promising strategy for cancer therapeutics could be exploring the antiangiogenic activity of the drug. ${ }^{23}$ The present study focused on investigating the developmental toxicity and antiangiogenic potentials of $M$. koenigii leaf extract in vivo. Zebrafish model system has gained much attention in recent years as an in vivo model for angiogenesis study due to its physiological similarities with mammals. ${ }^{24,25}$ The results of this study showed that the aqueous extract of $M$. koenigii leaves are quite safe and the deleterious effects on development are visible only at high concentrations of $1,500 \mathrm{ppm}$ and above. The developmental defects such as edema in heart, incomplete yolk sac absorption, and absence of swim bladder were observed in embryos when exposed to 2,000 ppm and above of $M$. koenigii extracts.

On the contrary, the ethanol extract appeared to be quite toxic and concentrations beyond $10 \mathrm{ppm}$ interfered with the survivability of embryos beyond $3 \mathrm{dpf}$. Severe developmental effects such as body curvature, yolk sac burst, tail bending, and pericardial edema were observed at all doses and none of the exposed embryos could survive till $5 \mathrm{dpf}$. The developmental deformities observed in the present study are in concordance with the previous studies on toxicity and teratogenic effects of natural products such as curcumin and celastrol. ${ }^{26,27}$

Characterization and screening of small molecules with antiangiogenic properties from natural sources have garnered wide attention in recent years. In this study, we have shown preliminary results of the effect of $M$. koenigii leaf extracts on vasculogenesis using transgenic zebrafish models. The results of this study indicated that the antiangiogenic activity of aqueous extract is more potent as compared with the ethanol extract. These observations corroborate the results shown in other studies conducted using natural products such as soya bean. ${ }^{28}$ From our data, it is not clear why the aqueous extract alone demonstrated antiangiogenic activities. Antioxidant activity of aqueous extracts of M. koenigii could contribute to our observations. Indeed, a study on rats has shown that the aqueous extract of curry leaf can alleviate the cadmium-induced oxidative damage in cardiac tissues through its antioxidant activity. ${ }^{29}$

\section{Conclusion}

Although the data presented here are preliminary, they, nevertheless, demonstrate that the crude aqueous extract of $M$. koenigii leaves is less toxic and possesses antiangiogenic properties. In contrast, the ethanol extract is more toxic and does not show any antiangiogenic activity. The study also highlights the usefulness of the zebrafish model system as an effective in vivo tool for screening such natural compounds for their anticancer activities. Future work should be directed toward isolating the active ingredients from this crude extract and characterizing the active ingredients for its anticancer properties using mammalian systems.

\section{Funding}

The financial support from Nitte (Deemed to be University) through the intramural research grant (NUSR2/2018/10/52) to D.D. and A.B. is gratefully acknowledged.

\section{Conflict of Interest}

None declared.

\section{References}

1 Ujowundu CO, Okafor OE, Agha NC, Nwaogu LA, Igwe KO, Igwe CU. Phytochemical and chemical composition of Combretum zenkeri leaves. J Med Plants Res 2010;4(10): 965-968

2 Handral HK, Pandith A, Shruthi SDA. Review on Murraya koenigii: multipotential medicinal plant. Asian J Pharm Clin Res 2012;5(4):5-14

3 Phumthum Ms, Balslev H. Thai ethnomedicinal plants used for diabetes treatment. OBM ICM 2018;3(3):1-25

4 Jianxin Z, Shuangkui D, Xuchang D, Shiheng Z. Effects of ultrahigh pressure processing on the physicochemical characteristics of Taibai Kudzu starch. Chin Soc Agric Eng 2007;23:269-271

5 Mackeen M, Ali A, El-Sharkawy S, et al. Antimicrobial and cytotoxic properties of some Malaysian traditional vegetables (ulam) Pharm Biol 1997;35(3):174-178

6 Goldshmit Y, Sztal TE, Jusuf PR. Hall TE, Nguyen-Chi M, Currie PD. Fgf-dependent glial cell bridges facilitate spinal cord regeneration in zebrafish. J Neurosci 2012;32(22):7477-7492

7 Mohan S, Abdelwahab SI, Cheah SC, et al. Apoptosis effect of girinimbine isolated fromMurraya koenigiion lung cancer cells in vitro. Evid Based Complement Alternat Med 2013;689865. Available at: https://www.hindawi.com/journals/ecam/2013/ $689865 /$

8 Kesari AN, Gupta RK, Watal G. Hypoglycemic effects of Murraya koenigii on normal and alloxan-diabetic rabbits. J Ethnopharmacol 2005;97(2):247-251

9 Hanahan D, Folkman J. Patterns and emerging mechanisms of the angiogenic switch during tumorigenesis. Cell 1996;86(3):353-364

10 Wang C, Tao W, Wang Y, et al. Rosuvastatin, identified from a zebrafish chemical genetic screen for antiangiogenic compounds, suppresses the growth of prostate cancer. Eur Urol 2010;58(3):418-426

11 Folkman J. Tumor angiogenesis: therapeutic implications. N Engl J Med 1971;285(21):1182-1186

12 Pandya NM, Dhalla NS, Santani DD. Angiogenesis-a new target for future therapy Vas. Pharmacol 2006;44(5):265-275

13 Isogai S, Horiguchi M, Weinstein BM. The vascular anatomy of the developing zebrafish: an atlas of embryonic and early larval development. Dev Biol 2001;230(2):278-301

14 Rubinstein AL. Zebrafish: from disease modeling to drug discovery. Curr Opin Drug Discov Devel 2003;6(2):218-223

15 Harbourne JB, Phytochemical Methods: A Guide to Modern Techniques of Plant Analysis. The Netherlands: Springer; 2005

16 Westerfield M, The Zebrafish Book: A Guide for the Laboratory Use of Zebrafish Eugene: University of Oregon Press; 2000

17 Kärber G. Beitrag zur kollektiven Behandlung pharmakologischer Reihenversuche. Naunyn Schmiedebergs Arch Exp Pathol Pharmakol 1931;162(4):480-483

18 Ko FN, Lee YS, Wu TS, Teng CM. Inhibition of cyclooxygenase activity and increase in platelet cyclic AMP by girinimbine, isolated from Murraya euchrestifolia. Biochem Pharmacol 1994;48(2):353-360 
19 Ito C, Itoigawa $\mathrm{M}$, Nakao $\mathrm{K}$, et al. Induction of apoptosis by carbazole alkaloids isolated from Murraya koenigii. Phytomedicine 2006;13(5):359-365

20 Nagappan T, Ramasamy P, Wahid ME, Segaran TC, Vairappan CS. Biological activity of carbazole alkaloids and essential oil of Murraya koenigii against antibiotic resistant microbes and cancer cell lines. Molecules 2011;16(11): 9651-9664

21 Hampl M, Tanaka T, Albert PS, Lee J, Ferrari N, Fine HA. Therapeutic effects of viral vector-mediated antiangiogenic gene transfer in malignant ascites. Hum GeneTher 2001;12(14): 1713-1729

22 Westermarck J, Kähäri VM. Regulation of matrix metalloproteinase expression in tumor invasion. FASEB J 1999;13(8):781-792

23 Carmeliet P, Jain RK. Angiogenesis in cancer and other diseases. Nature 2000;407(6801):249-257

24 Bakkiyanathan A, Nathan JR, Ravikumar S, Gopalakrishnan TS, Aruldas FM, Malathi R. Anti-angiogenic effects of theophylline on developing zebrafish (Danio rerio) embryos. Biomed Prevent Nutr 2012;2(3):174-178

25 Kidd KR, Weinstein BM. Fishing for novel angiogenic therapies. Br J Pharmacol 2003;140(4):585-594

26 Wu JY, Lin CY, Lin TW, Ken CF, Wen YD. Curcumin affects development of zebrafish embryo. Biol Pharm Bull 2007;30(7):1336-1339

27 Wang S, Liu K, Wang X, He Q Chen X. Toxic effects of celastrol on embryonic development of zebrafish (Danio rerio) Drug Chem Toxicol 2011;34(1):61-65

28 Bakkiyanathan A, Joseph AM, Tharani L, Malathi R. Genistein, the phytoestrogen induces heart-and-soul (has) phenotypes in zebrafish embryo. J Dev Biol Tissue Eng 2010;2(2):18-22

29 Mitra E, Ghosh AK, Ghosh D, et al. Protective effect of aqueous curry leaf (Murraya koenigii) extract against cadmium-induced oxidative stress in rat heart. Food Chem Toxicol 2012;50(5):1340-1353 